\title{
A COMPREENSÃO DE LEITURA TEXTUAL COMO UM INSTRUMENTO DE DIAGNÓSTICO DE PACIENTES COM DEMÊNCIA DE ALZHEIMER LEVE E MODERADA1
}

\author{
Juciclara Rinaldi² \\ Marília Silveira ${ }^{3}$ \\ Renata Kochhann ${ }^{4}$ \\ Maria Alice de Mattos Pimenta Parente 5
}

\section{Resumo}

A tarefa de reconto de histórias apresenta diferença entre idosos sem e com demência, pois aqueles lembram maior número de informações do que estes. Com o objetivo de verificar se, desde as etapas iniciais, os pacientes com Demência de Alzheimer (DA) leve e moderada se mostram sensíveis à tarefa de compreensão de leitura textual, analisaram-se 40 idosos: 25 com DAs prováveis, sendo 12 leves e 13 moderados; e 15 sem demência, com escolaridade de 4 anos ou mais. Os pacientes foram avaliados através do Mini-Exame do Estado Mental (MEEM), Questionário Sociodemográfico, Escala Reduzida de Depressão Geriátrica da Yesavage (GDS-15) e quatro histórias, com quatro frases cada, as quais apresentavam estrutura textual descritiva e narrativa. A compreensão textual mostrou ser uma tarefa sensível aos participantes com demência, cujo desempenho foi significativamente pior do que o dos idosos saudáveis. Não houve diferença estatisticamente significativa quanto às estruturas textuais das histórias. Contudo, à medida que aumentaram os elementos de estrutura narrativa nas histórias, os participantes sem demência e os com demência leve apresentaram melhora na recordação. Os tipos de acréscimos às histórias mais freqüentes no grupo com DA foram reconstrução e inferência. Os resultados sugerem que a ava-

1 Estudo derivado da Dissertação "Influência do Hipertexto na Compreensão Textual de Pacientes com Demência de Alzheimer Leve e Moderada”, apresentada em 2007 ao Curso de Pós-Graduação em Psicologia do Desenvolvimento do Instituto de Psicologia da Universidade Federal do Rio Grande do Sul (UFRGS), pela primeira autora sob orientação da última.

2 Psicóloga. Mestre em Psicologia pela UFRGS. E-mail: juciclara@terra.com.br

3 Estudante de Psicologia da Universidade do Vale do Rio dos Sinos (Unisinos). E-mail: marilia.unisinos@ gmail.com

4 Estudante de Psicologia da Unisinos. E-mail: renata_kochhann@yahoo.com.br

5 Doutora em Psicologia pela Universidade de São Paulo (USP). Professora Adjunta do Departamento de Psicologia do Desenvolvimento e da Personalidade e do Programa de Pós-Graduação em Psicologia do Instituto de Psicologia da UFRGS. E-mail: malicemp@terra.com.br

Estud. interdiscip. envelhec., Porto Alegre, v. 13, n. 1, p. 117-132, 2008. 
liação das habilidades lingüísticas pode auxiliar no diagnóstico diferencial precoce da Demência.

Palavras-chave: Demência de Tipo Alzheimer. Compreensão textual. Memória. Linguagem.

\section{Introdução}

Além do declínio de memória, a linguagem também tem seu desempenho afetado nas primeiras fases da Demência de Alzheimer (DA). Uma das tarefas de linguagem que se encontra prejudicada logo neste período é a compreensão de leitura textual. Ela exige várias habilidades lingüísticas, como decodificação da linguagem impressa, compreensão das palavras informadas e a reconstrução e organização de reconto mediante a expressão da voz do falante, no caso de a história ser lida em voz alta (APPELL; KERTESZ; FISMAN, 1982; MILLER, 1989; MANSUR et al., 2005).

Além disso, para compreender um texto, são necessários vários processos cognitivos funcionando conjuntamente. Dentre eles encontram-se a memória de trabalho, cuja função é armazenar o estímulo por tempo suficiente para processá-lo e interpretá-lo, e as memórias semânticas e episódicas, oferecendo sentido ao que está sendo narrado (MATLIN, 2004; SQUIRE; KANDEL, 2003). As dificuldades de memória que ocorrem precocemente na DA dificultam a compreensão textual. Nas primeiras etapas da patologia, a memória episódica e a memória de trabalho apresentam declínio evidente; contudo, a memória semântica mantém-se funcional por mais tempo.

Por ser uma tarefa complexa, a compreensão de leitura textual e seu reconto envolvem também recursos atencionais. Um estudo realizado por Berardi, Parasuraman e Haxby (2005), concluiu que pacientes com DA provável, em estágio leve, apresentam diminuída capacidade de vigilância. Sabe-se que a atenção seletiva, especificamente, os processos inibitórios estão prejudicados precocemente (PIGNATTI et al., 2005). A atenção dividida declina posteriormente (BADDELEY et al., 2001) enquanto que a atenção sustentada é o último componente a apresentar deficit (PERRY; HODGES, 1999).

Trabalhos como os de Braver et al. (2005), Chapman et al. (2002), Gély-Nargeot, Ska, e Touchon (2002) e Parente et al. (2005), de mesma forma, salientam que idosos sem demência lembram maior número de informações do que os com demência. Nesse sentido, provas de reconto de histórias são instrumentos eficazes para discriminar a patologia (ORANGE; KERTESZ, 2000). 
Estudos em pacientes com DA têm evidenciado que o tipo de estrutura textual pode facilitar a compreensão, como o estudo comparativo entre pacientes com DA e idosos sem demência, no qual os participantes deveriam recontar quatro histórias, duas narrativas e duas descritivas (GÉLYNARGEOT; SKA; TOUCHON, 2002). Os autores observaram que tanto os pacientes com DA quanto os sem demência mostraram-se sensíveis à estrutura textual. A compreensão de todos os participantes foi melhor quando a estrutura era de uma narrativa. Esses autores também verificaram que versões mais detalhadas, ou seja, mais redundantes, melhora a compreensão dos pacientes com DA. Em outros dois estudos, os achados demonstraram que a recordação, tanto da idéia principal quanto dos detalhes, decresce com o agravamento da demência. Entretanto, apesar dessa piora geral, as idéias principais permanecem mantidas por mais tempo do que os detalhes. Portanto, o agravamento da demência, além de prejudicar a recordação de história dos pacientes com demência, faz com que eles privilegiem a idéia principal do texto em detrimento dos detalhes (HUDON et al., 2006; JOHNSON; STORANDT; BALOTA, 2003).

Uma vez que a estrutura textual influencia a compreensão do texto de pacientes com DA, a manipulação de seus componentes pode auxiliar os pacientes nessa tarefa. Labov e Waletzky (1967) apresentam um modelo estrutural de narrativas. Este modelo sugere cinco macrocategorias na composição da estrutura global de uma narrativa: orientação ou situação, complicação, avaliação, resolução e conclusão ou moral (FÁVERO, 2002; VIEIRA, 2001). A narrativa inicia-se com a macrocategoria orientação ou situação, que informa o leitor quanto a lugar, tempo, pessoa e situação. Segue a macrocategoria denominada complicação, que apresenta uma série de eventos, servindo para desenvolver a ação nas narrativas. A avaliação faz a comparação de algumas unidades narrativas com outras, dentro da mesma estrutura. A macrocategoria resolução encerra as ações. Algumas narrativas chegam à conclusão da história - macrocategoria opcional da moral - oferecendo uma síntese do ocorrido.

Apesar de os textos poderem ser classificados quanto à sua estrutura como descrição, narração e argumentação, normalmente essas três categorias ocorrem num mesmo texto. Um autor (ou narrador) pode ater-se mais a uma dessas etapas, qualificando determinadas ocorrências e tornando suas informações mais redundantes. Assim, se o texto contiver apenas a orientação/situação ou um grande número de frases nessa categoria, ele terá uma aparência mais descritiva. Por outro lado, se o autor relatar de forma 
equivalente quatro ou cinco das macrocategorias, seu texto será considerado uma narrativa.

Este estudo analisará a compreensão de leitura textual de pacientes com DA, através do reconto de histórias. O objetivo do presente estudo é de verificar se, desde as etapas iniciais, os pacientes com DA leve e moderada se mostram sensíveis à tarefa de compreensão de leitura textual. As quatro histórias serão avaliadas através do número de proposições recordadas nos textos, sendo caracterizadas como mais descritivas ou mais narrativas, pois, segundo Gély-Nargeot, Ska e Touchon (2002), um tipo de estrutura favorece a recordação.

\section{Método}

A seguir estão descritos os participantes do estudo e as escalas utilizadas para selecioná-los dentro de cada grupo. Também seguem descrição dos materiais e procedimentos utilizados neste trabalho.

\subsection{Participantes}

Participaram 40 idosos (14 homens e 26 mulheres) reunidos em três grupos: 12 idosos com DA leve; 13 com DA moderada; e 15 controles. Doze dos 25 sujeitos com DA residiam em suas casas e 13 em instituições; 12 dos 15 controles residiam em seus lares e três em instituições.

Foram incluídos pacientes com DA provável, conforme critérios do National Institute of Neurological Comunicative Disorders and StrokeAlzheimer's Disease and Related Disorders (NINCDS-ADRDA), sendo:

a) deficit em duas ou mais áreas da cognição;

b) piora das funções cognitivas e outras áreas da cognição;

c) ausência de distúrbio da consciência severo;

d) início entre os 40 e 90 anos, sendo mais freqüente após os 65 anos;

e) exames clínicos e laboratoriais que apóiem o diagnóstico, além da confirmação pelo Mini-Exame do Estado Mental (MEEM), Escala de Blessed e testes neuropsicológicos;

f) ausência de doenças sistêmicas ou outras doenças cerebrais que por si só possam provocar declínio progressivo de memória e cognição (McKHANN et al., 1984).

No Diagnostic and Statistical Manual of Mental Disorders, Fourth Edition, Text Revision (DSM-IV-TR), primeiro os critérios apontam para o 
aparecimento de prejuízos de memória e de perturbações cognitivas como a afasia, a apraxia e a agnosia. Segundo, aparece deficit no funcionamento executivo que, torna os aspectos social e ocupacional da vida do paciente muito difícil de administrar, com escolaridade mínima de quatro anos. Os participantes possuíam diagnóstico médico de Demência de Alzheimer realizado por seus médicos. Os participantes com DA foram alocados em dois grupos, de acordo com o estágio da demência, ou seja, DA leve e DA moderada conforme escores do MEEM, que variou de 12 a 24 pontos, sendo entre 12 e 18 pontos para os pacientes com DA moderada; os participantes com DA leve que foram incluídos no estudo obtiveram pontuação entre 19 e 24 pontos. Para ambos os grupos com DA a pontuação da Escala Reduzida de Depressão Geriátrica da Yesavage (GDS-15) (ALMEIDA; ALMEIDA, 1999) foi até 10 pontos. O grupo controle foi emparelhado quanto a idade e a escolaridade com os participantes com DA. Eles possuíam escore do GDS-15 < 10 pontos, MEEM maior que 24 pontos e escolaridade mínima de quatro anos. Não foram incluídos os participantes com problemas psiquiátricos, exceto com sintomas depressivos até o estágio moderado, problemas visuais ou auditivos não corrigidos. O projeto foi aprovado pelo Comitê de Ética em Pesquisa da Universidade Federal do Rio Grande do Sul (UFRGS) sob número 2006574. Cada participante assinou o Termo de Consentimento Livre e Esclarecido, conforme Resolução 16 do Conselho Federal de Psicologia (BRASIL, 2000) para pesquisa com seres humanos. Na Tabela 1 está demonstrada a caracterização da amostra.

Tabela 1 - Caracterização da amostra nos três grupos.

\begin{tabular}{|c|c|c|c|c|}
\hline \multirow{2}{*}{ Variáveis } & DA leve & DA moderada & Controle & \multirow{2}{*}{$\mathbf{p}^{*}$} \\
\hline & M (DP) & M (DP) & M (DP) & \\
\hline Escolaridade (anos) & $8,18^{a}(3,22)$ & $7,67 \mathrm{a}(3,31)$ & $8,87 \mathrm{a}(3,50)$ & NS \\
\hline Idade (anos)** & $76,25^{\mathrm{a}}(4,07)$ & $78,67 \mathrm{a}(4,52)$ & $76,53^{a}(4,95)$ & NS \\
\hline \multirow[t]{2}{*}{ MEEM } & $21,41^{\mathrm{a}}(1,50)$ & $15,46^{\mathrm{b}}(1,66)$ & $27,53^{\text {с }}(1,88)$ & $<0,001$ \\
\hline & n (\%) & n (\%) & n (\%) & \\
\hline Sexo masculino & $6(50 \%)$ & $4(30,8 \%)$ & $4(26,7 \%)$ & NS \\
\hline \multirow[t]{2}{*}{ Estado civil viúvo } & $5(50 \%)$ & $7(53,8 \%)$ & $5(33,3 \%)$ & NS \\
\hline & Mediana (AIQ) & Mediana (AIQ) & Mediana (AIQ) & \\
\hline GDS-15 & $3(2,25 \text { a } 6)^{\mathrm{a}}$ & $4(3 \text { a } 5,75)^{\mathrm{a}}$ & $2(1 \mathrm{a} 4)^{\mathrm{a}}$ & NS \\
\hline
\end{tabular}

Estud. interdiscip. envelhec., Porto Alegre, v. 13, n. 1, p. 117-132, 2008. 
* Diferença não significativa ( $>>0,05)$ de acordo com o teste t para amostras emparelhadas e o teste ANOVAOneway para calcular o GDS-15.

** Não coletado para o grupo controle.

\subsection{Instrumentos e Materiais}

Após a assinatura do Termo de Consentimento Livre e Esclarecido, foram aplicados, para todos os sujeitos o MEEM (FOLSTEIN; FOLSTEIN; McHUGH, 1975) adaptado para Porto Alegre por Chaves e Izquierdo (1992), o Questionário Sociodemográfico, e o GDS-15 (YESAVAGE et al., 1983) adaptado para o Brasil por Almeida e Almeida (1999). Por fim, as quatro histórias foram aplicadas. Uma ordem aleatória foi estabelecida para não ocorrer o efeito de ordem. A avaliação ocorreu em um único encontro, com duração de uma hora e trinta minutos, em média.

Um instrumento computadorizado, utilizando o programa E-Prime, foi construído especificamente para a apresentação de quatro histórias, cada uma com quatro frases. Elas foram criadas para este estudo e referiam-se a diferentes temas: professor, jogador de futebol, cachorro e amigo. A história do professor continha apenas frases da macrocategoria situação/orientação, apresentando estruturas textuais de história descritiva; a do jogador de futebol contemplou as macrocategorias situação e complicação; a do cachorro situação, complicação e resolução; e a do amigo, apresentou situação, complicação, resolução e conclusão, caracterizando uma narrativa. A Figura 1 mostra as quatro histórias lineares com as respectivas macrocategorias.

\begin{tabular}{|c|c|}
\hline \multicolumn{2}{|c|}{ Histórias Lineares } \\
\hline $\begin{array}{l}\text { Professor } \\
\text { O professor era inteligente } \\
\text { Ele ensinava na universidade } \\
\text { Suas aulas eram divertidas } \\
\text { E ele era muito paciente }\end{array}$ & $\begin{array}{l}\text { Jogador de Futebol } \\
\text { O jogador de futebol era talentoso } \\
\text { Um dia ele se machucou } \\
\text { Por isso, ele precisou fazer exercícios difí- } \\
\text { ceis } \\
\text { Todos no time o admiravam por isso }\end{array}$ \\
\hline Orientação & Orientação Complicação \\
\hline $\begin{array}{l}\text { Cachorro } \\
\text { O cachorro era um vira-lata } \\
\text { Desde pequeno ele vivia nas ruas } \\
\text { Uma manhã ele acordou com muita fome } \\
\text { Então foi procurar comida na vizinhança }\end{array}$ & $\begin{array}{l}\text { Meu Amigo } \\
\text { Meu amigo estava bravo } \\
\text { Ao meio dia sua esposa não tinha feito o almo- } \\
\text { ço } \\
\text { E ele ia se atrasar para o trabalho } \\
\text { Então resolveram almoçar no restaurante da } \\
\text { esquina }\end{array}$ \\
\hline Orientação Complicação Resolução & Orientação Complicação Resolução Conclusão \\
\hline
\end{tabular}

Figura 1: Histórias e suas respectivas macrocategorias. 
Inicialmente, na tela do computador com monitor colorido, de 17 polegadas, foram apresentadas explicações sobre como proceder para construir a história. Após as explicações iniciou-se a apresentação da história que seguem a seqüência ilustrada na Figura 2.

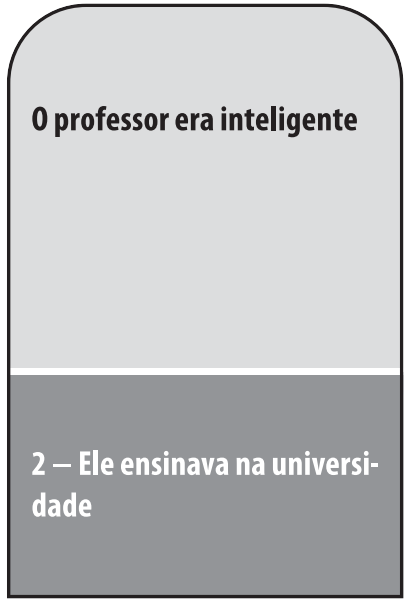

1a Tela

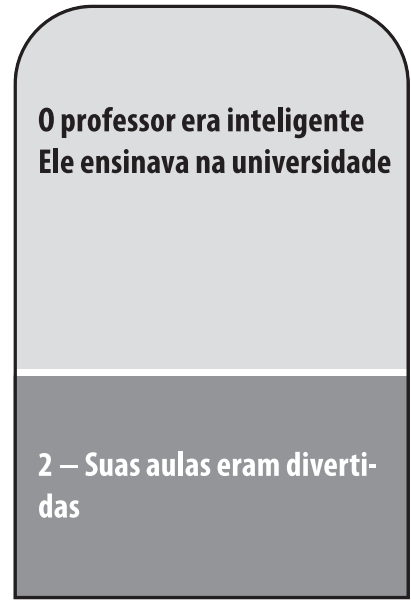

$2^{a}$ Tela

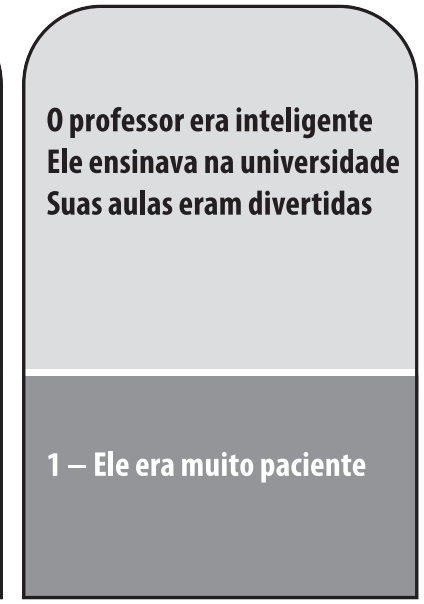

$3^{\text {a Tela }}$

Figura 2: Seqüência de telas da história do professor (ver explicação no texto).

Observa-se, na primeira tela da Figura 2, que a primeira frase da história aparecia na parte superior da tela. Esta parte apresentava um fundo azul claro com as letras brancas. Na parte inferior da tela, estava a frase que dava continuidade à história: "Ele ensinava na universidade”. A parte inferior da tela o fundo era azul escuro com letras na cor amarela. Uma linha branca dividia a tela em duas partes. O participante lia tanto a frase que iniciava a história, quanto a que aparecia para continuá-la, em voz alta. A ação de clicar o número da frase trocava a tela do computador, surgindo a frase selecionada na parte superior da tela. Simultaneamente, uma nova opção de frase, "Suas aulas eram divertidas”, apareceria na parte inferior da tela do computador, substituindo a anterior (Figura 2, segunda tela). Esta seqüência continuava até as quatro frases da história estarem na parte superior da tela. Ao final da construção, os participantes liam em voz alta a história construída, uma única vez. O reconto era gravado; e o conteúdo recordado, transcrito para análise.

A avaliação do reconto dos participantes foi realizada através de proposições, unidades de significado que se dão através da relação entre 
dois elementos do texto (KINTSCH; VAN DIJK, 1978). O percentual entre o número de proposições lembradas e o total de proposições da história original resultou no escore da recordação, em percentual. Os participantes leram as histórias em voz alta, uma única vez.

Na presente pesquisa, os acréscimos às histórias, ou seja, proposições do reconto dos participantes que não se encontravam na história original, foram classificados em três categorias (PARENTE; CAPUANO; NESPOULOUS, 1999):

a) reconstrução: palavras ou expressões que não constavam na história lida, entretanto apareciam no reconto da história;

b) interferência: modificação do significado da história devido a junção em uma mesma proposição de dois elementos originalmente encontradas em duas proposições diferentes;

c) inferência: comentários sobre fatos ou eventos relacionados com a história original, que não se encontravam na superfície textual da mesma, e que, contudo, não alteraram o sentido.

A concordância entre as juízas obteve 95\% de concordância em todas as comparações das histórias do professor e do amigo. Nas outras duas histórias (cão e jogador de futebol) esses escores foram obtidos em pelo menos uma das comparações. Nas ocasiões em que houve discordância, foi realizada uma discussão da avaliação do percentual de lembrança da história até um acordo.

\section{Resultados}

A Tabela 2 apresenta as médias (M) e desvios-padrão (DP) das proposições lembradas, por grupo, nas diferentes histórias. A comparação entre os três grupos, através do teste da ANOVA-Oneway, apresentou diferença significativa entre os três grupos nos percentuais de lembranças de todas as histórias ( $\mathrm{p}<0,001)$. Conforme esperado, o grupo controle foi o que mais lembrou das proposições nas cinco histórias, seguido do grupo DA leve e DA moderada, respectivamente.

Os dois grupos de participantes com DA apenas não diferiram significativamente na história do cachorro, ou seja, na que finalizou na macrocategoria resolução $(\mathrm{p}=0,116)$, em vista de um desempenho um pouco melhor dos participantes com DA moderada, com relação a lembrança das demais histórias deste grupo. O grupo controle e o grupo DA leve apresentaram um desempenho em curva U nas histórias, sendo que a história descritiva (professor/situação) e a com todos os elementos da narrativa (amigo/conclusão) 
obtiveram um escore mais alto do que as histórias do jogador (complicação) e a do cachorro (resolução).

Tabela 2 - Percentual de lembrança das histórias.

\begin{tabular}{|c|c|c|c|c|c|c|c|c|c|}
\hline \multirow{2}{*}{ Histórias } & \multicolumn{2}{|l|}{ DA leve } & \multicolumn{2}{|c|}{ DA moderada } & \multicolumn{2}{|l|}{ Controle } & \multicolumn{2}{|l|}{ Total } & \multirow{2}{*}{$\mathbf{p}^{*}$} \\
\hline & M (DP) & $\mathbf{n}$ & M (DP) & n & M (DP) & $\mathbf{n}$ & M (DP) & $\mathbf{n}$ & \\
\hline Professor & $60,71(18,40)^{a}$ & 12 & $33,77(24,14)^{b}$ & 11 & $83,67(11,00)$ c & 14 & $61,39(27,12)$ & 37 & $<0,001$ \\
\hline Jogador & $46,79(21,87)^{a}$ & 12 & $21,30(21,10)^{b}$ & 13 & $80,51(11,94)^{c}$ & 15 & $51,15(30,94)$ & 40 & $<0,001$ \\
\hline Cachorro & $50,69(20,86)^{\mathrm{a}}$ & 12 & $37,50(18,63)^{\mathrm{a}}$ & 12 & $80,35(13,32)^{b}$ & 14 & $57,46(25,24)$ & 38 & $<0,001$ \\
\hline Amigo & $55,37(25,86)^{a}$ & 11 & $21,97(21,39)^{\mathrm{b}}$ & 12 & $82,42(14,36) \mathrm{c}$ & 15 & $55,50(32,45)$ & 38 & $<0,001$ \\
\hline
\end{tabular}

Fonte: Dados obtidos na pesquisa.

Notas: $\mathrm{M}$ = média; $\mathrm{DP}$ = desvio-padrão; letras iguais (a, b, c) representam valores iguais.

* Diferença significativa $(\mathrm{p}<0,001)$.

Nas histórias lineares do professor, do jogador e do amigo, os participantes com DA leve lembraram um maior número de proposições do que o grupo com DA moderada, sendo essa diferença estatisticamente significativa ( $\mathrm{p}=0,006, \mathrm{p}=0,007$ e $\mathrm{p}=0,003$ respectivamente). Apesar de uma melhor lembrança dos pacientes com DA leve, se comparados com os de DA moderado, a diferença não obteve valor significativo.

Ao observar qualitativamente o reconto, verificou-se que o grupo controle obedeceu à seqüência do texto original. Os idosos com DA leve lembraram o tema e alguns detalhes da história. Já o grupo com DA moderada lembrou o tema da história sem detalhes.

Agrupando-se os escores das duas histórias lineares com características mais descritivas (professor/situação e jogador/complicação) e das duas histórias lineares com características mais narrativas (cachorro/resolução e amigo/conclusão), novamente não foram observadas diferenças significativas. Isso ocorreu pela maior lembrança nas histórias que finalizaram em complicação e em resolução, ou seja, macrocategorias em posição intermediária quanto às características estruturais de uma narrativa. Os acréscimos (reconstruções, inferências e interferências) para cada história realizada por cada grupo são apresentados na Tabela 3. 
Tabela 3 - Tipos de acréscimos por grupo em cada história.

\begin{tabular}{lcccc}
\hline Tipos de & DA Leve & DA Moderada & Controle & Total \\
\cline { 2 - 5 } Acréscimos & $\mathbf{n}$ & $\mathbf{n}$ & $\mathbf{n}$ & $\mathbf{n}$ \\
\hline Interferência & 4 & 2 & 1 & 7 \\
Inferência & 15 & 10 & 4 & 29 \\
Reconstrução & 16 & 14 & 4 & 34 \\
Total & 35 & 26 & 9 & 70 \\
\hline
\end{tabular}

Fonte: Dados obtidos na pesquisa.

Notas: DA = Demência de Alzheimer; $\mathrm{n}$ = número de ocorrências.

Não houve diferença significativa de acréscimos entre os grupos. Contudo, há uma tendência do grupo com DA leve em realizar acréscimos quando observados os demais grupos. Esta aparente vantagem dos pacientes moderados pode decorrer do fato dos acréscimos serem considerados em seus escores brutos e não em porcentagens. Como as produções dos pacientes com DA leve foram mais abundantes, também, encontrou-se um maior número de acréscimos. Em contrapartida, observou-se menor número de acréscimos para o grupo controle, grupo este com maior número de proposições, do que para os grupos com DA leve e com DA moderada em todas as histórias. O grupo com DA leve apresentou maior número de acréscimos nas histórias lineares do professor, jogador de futebol e amigo. O grupo com DA moderada apresentou maior número de acréscimos na história do cachorro.

\section{Discussão}

A comparação entre os três grupos do estudo apresentou diferença estatisticamente significativa nos percentuais de lembrança de todas as histórias, sendo maior para o grupo controle, seguido do grupo com DA leve e por último os participantes com DA moderada. Nesse sentido, provas de reconto de histórias são instrumentos eficazes para discriminar o envelhecimento normal daquele associado com a DA (ORANGE; KERTESZ, 2000). Estudos sobre reconto de histórias demonstram que idosos sem demência lembram maior número de informações do que os com demência (BRAVER et al., 2005; CHAPMAN et al., 2002; GÉLY-NARGEOT; SKA; TOUCHON, 2002; PARENTE et al., 2005; GÓMEZ; WHITE, 2006). Isso demonstra que é possível realizar o diagnóstico diferencial entre o envelhecimento saudável e o início da demência combinando a avaliação da fluência verbal com o 
reconto de história. O presente estudo confirma que falhas de compreensão textual em idosos podem ser indícios de processos demenciais, mesmo no caso de histórias bastante curtas como as aqui utilizadas. Assim, o recontar de histórias pode ser inserido numa bateria de avaliação de idosos, sem necessariamente despender muito tempo e provocar cansaço ao paciente.

Foram encontradas, também, diferenças entre os dois grupos de pacientes com DA. O grupo com DA moderada lembrou-se menos proposições do que o grupo com DA leve. Ainda, o padrão de recordação dos dois grupos apresentou diferença: os DAs leves lembraram da idéia principal e de alguns detalhes das histórias, enquanto que os DAs moderados lembraram-se apenas do personagem principal ou da ação. Já foi observado por Johnson, Storandt e Balota (2003) e por Hudon et al. (2006) que a recordação dos pacientes com demência privilegia a idéia principal de texto em detrimento dos detalhes. Essas dificuldades podem decorrer do fato de que, com a piora da atenção no estágio moderado da demência, há dificuldade de manutenção das informações, prejudicando o desempenho da memória de trabalho (BERARDI; PARASURAMAN; HAXBY, 2005; JOHNSON; STORANDT; BALOTA, 2003; PERRY; HODGES, 1999).

Por outro lado, o tipo de estrutura das narrativas não influenciou a recordação, uma vez que o reconto das histórias que representavam narração e descrição não diferiram significativamente. Desta forma, nosso estudo não corrobora a pesquisa de Gély-Nargeot, Ska e Touchon (2002), que encontraram diferença significativa entre as duas modalidades, tendo favorecido para recordação dos DAs a história que caracterizava uma narrativa. A ausência de diferença estatística no presente trabalho pode ter ocorrido devido a:

a) grande redundância na história descritiva;

b) pouca extensão das histórias criadas.

Gély-Nargeot, Ska e Touchon (2002), acreditam que a melhora de seus pacientes na recordação de narrativas, em relação à recordação de descritivas, pode ser devido ao grande número de idéias redundantes, pois as primeiras contêm maior número de redundâncias do que as últimas. Levantam a hipótese de que as descrições que envolvem número de informações diferentes tornam sua memorização mais árdua quando comparada à narrativa, cuja seqüência de ações possibilita encadeamento de idéias, facilitando o armazenamento na memória. No entanto, a história descritiva elaborada nesse estudo (professor), ao contrário, apresentou maior grau de redundância de idéias, pois as quatro frases relatavam qualidades que enfatizam suas características virtuosas. 
Por outro lado, ao analisar as histórias com uma estrutura mínima de narrativa, ou seja, aquelas que apresentam dois elementos de macrocategorias ou mais, observou-se que as histórias do jogador (situação e complicação) e do cachorro (situação, complicação e resolução) apresentaram recordação de $46,79 \%$ e $50,69 \%$, respectivamente, sendo um percentual menor que a do amigo (situação, complicação, resolução e conclusão), que foi de 55,37\%. Pode-se pensar que o aumento de elementos da narrativa auxiliou a lembrança de idosos sem demência e aqueles com demência leve, indicando que a estrutura da narrativa ajuda na associação de idéias. Os elementos da narrativa, bem como a redundância, auxiliam a coesão e a coerência de uma história, favorecendo sua recordação.

Nesta pesquisa, a ocorrência de acréscimos na lembrança das histórias salienta a dificuldade dos participantes com DA na recordação das informações lidas. O tipo de acréscimo que os grupos com DA privilegiaram foi: na DA leve e moderada, reconstrução e inferência. Os sujeitos com demência leve e moderada realizaram inferências, isto é, comentários sobre fatos e eventos relacionados com a história original não-presentes na superfície textual. Ambos os grupos acrescentaram palavras ou expressões que não constavam na história construída ou lida. Segundo Van Dijk (2004), a compreensão de um texto ocorre com a interpretação da informação lida através do conhecimento mais geral que possuímos. No presente estudo, os participantes com DA podem ter utilizado o subsistema de memória semântica, por apresentarem uma redução acentuada dos recursos da memória de trabalho e da memória episódica (PIGNATTI et al., 2005).

\section{Conclusão}

No presente estudo, ficou evidente que há diferença na lembrança dos idosos saudáveis, dos com DA leve e dos com DA moderada. A avaliação da compreensão de histórias auxilia o diagnóstico diferencial precoce da Demência. Porém, são necessárias outras pesquisas para se certificar sobre a influência da estrutura textual no desempenho dos pacientes com DA, pois os resultados contrários a essa hipótese podem ser decorrentes da diminuída extensão das histórias, minimizando diferenças entre as estruturas descritiva e narrativa. Entretanto, a pequena extensão das sentenças das histórias apresentadas favorece a avaliação para fins de diagnóstico por serem de rápida aplicação. 


\section{TEXT READING COMPREHENSION AS A TOOL FOR THE DIAGNOSIS OF PATIENTS WITH MILD AND MODERATE ALZHEIMER'S DISEASE}

\section{Abstract}

The task of recall stories presents a difference between the elderly with dementia and the ones without dementia because the first can remember a greater number of information than the latter. This study aimed to verify if patients with mild or moderate dementia are sensible to the task of textual reading comprehension since the first stages of the disease 40 elderly patients were analyzed: 25 with probable $\mathrm{AD}$, from which 12 were mild and 13 were moderate, and 15 without dementia, with a minimum schooling of 4 years. The patients were evaluated through the MEEM, Sociodemographic Questionary and the GDS-15, besides four stories, each with four sentences, which presented a narrative and descriptive textual structure. The textual comprehension showed to be a sensitive task to the patients with dementia, whose performance was significantly worse than the one of the healthy elderly. There was no statistically significant difference regarding the textual structures of the stories. However, as the elements of narrative structure in the stories increased, the participants without dementia and the ones with mild dementia showed an improvement in remembering the stories. The kinds of additions to the stories that the patients with AD made more frequently were reconstruction and inference. The results suggest that the evaluation of the linguistic abilities may help in the early differential diagnosis of dementia.

Keywords: Alzheimer's disease. Textual comprehension. Memory. Language.

\section{REFERÊNCIAS}

ALMEIDA, Osvaldo; ALMEIDA, Shirley. Confiabilidade da Versão Brasileira da Escala de Depressão em Geriatria (GDS): versão reduzida. Arquivos Neuropsiquiátricos, São Paulo, v. 57, n. 2-b, p. 421-426, 1999.

APPELL, Julian; KERTESZ, Andrew; FISMAN, Michael. A Study of Language Functioning in Alzheimer patients. Brain and Language, New York, v. 17, n. 1, p. 73-91, 1982. 
BADDELEY, Alan et al. Attentional Control in Alzheimer's Disease. Brain, London, v. 124, p. 1492-1508, 2001.

BERARDI, Anna Maria; PARASURAMAN, Raja; HAXBY, James. Sustained Attention in Mild Alzheimer's Disease. Developmental Neuropsychology, Mahwah, v. 28, n. 1, p. 507-537, 2005.

BRAVER, Todd et al. Context Processing and Context Maintenance in Healthy Aging and Early Stage Dementia of the Alzheimer's Type. Psychology and Aging, Arlington, v. 20, n. 1, p. 33-46, 2005.

BRASIL. Conselho Federal de Psicologia. Resolução CFP n. 16, de 20 de Dezembro de 2000. Dispõe sobre a realização de pesquisa em Psicologia com seres humanos. Brasília, DF, 2000. Disponível em: <http://www.pol. org.br/legislacao/doc/resolucao2000_16.doc>. Acesso em: 20 jan. 2008.

CHAPMAN, Sandra et al. Discourse Changes in Early Alzheimer Disease, Mild Cognitive Impairment, and Normal Aging. Alzheimer Disease and Associated Disorders, Hagerstown, v. 16, n. 3, p. 177-186, 2002.

CHAVES, Márcia; IZQUIERDO, Ivan. Differential Diagnosis Between Dementia and Depression: a study of efficiency increment. Acta Neurologica Scandinavica, Copenhagen, v. 85, n. 6, p. 378-382, 1992.

FÁVERO, Leonor. Coesão e Coerência Textuais. São Paulo: Ática, 2002.

FOLSTEIN, Marshal; FOLSTEIN, Susan; McHUGH, Paul. Mini-Mental State: a practical method for grading the cognitive state of patients for the clinician. Journal of Psychiatric Research, Oxford, v. 12, n. 3, p. 189-198, 1975.

GÉLY-NARGEOT, Marie Christine; SKA, Bernardette; TOUCHON, Jacques. Text Structure and Content Modulate the Recall of Patients with Dementia of the Alzheimer's Type. Brain and Cognition, Oxford, v. 48, n. 2/3, p. 371-375, 2002.

GÓMEZ, Rowena; WHITE, Desirée. Using Verbal Fluency to Detect Very Mild Dementia of the Alzheimer Type. Archives of Clinical Neuropsychology, New York, v. 21, n. 8, p. 771-775, 2006. 
HUDON, Carol et al. Memory for Gist and Detail Information in Alzheimer's Disease and Mild Cognitive Impairment. Neuropsychology, Washington, DC, v. 20, n. 5, p. 566-577, 2006.

JOHNSON, David; STORANDT, Martha; BALOTA, David. Discourse Analysis of Logical Memory Recall in Normal Aging and in Dementia of the Alzheimer Type. Neuropsychology, Washington, DC, v. 17, n. 1, p. 82-92, 2003.

KINTSCH, Walter; VAN DIJK, Teun. Toward a Model of Text Comprehension and Production. Psychological Review, Washington, DC, v. 85, n. 5, p. 363-394, 1978.

LABOV, William; WALETZKY, Joshua. Narrative Analysis: oral versions of personal experience. In: HELM, J. Essays on the Verbal and Visual Arts. Seattle: University of Washington Press, 1967. p. 12-44.

MANSUR, Letícia et al. Linguagem e Cognição na Doença de Alzheimer. Psicologia: Reflexão e Crítica, Porto Alegre, v. 18, n. 3, p. 300-307, 2005.

MATLIN, Margaret. Psicologia Cognitiva. Rio de Janeiro: LTC, 2004.

McKHANN, Guy et al. Clinical Diagnosis of Alzheimer's Disease: report of the NINCDS-ADRDA Work Group under the auspices of Departament of Health and Human Services Task Force on Alzheimer's Disease. Neurology, New York, v. 34, n. 7, p. 939-944, 1984.

MILLER, Edgar. Language Impairment in Alzheimer Type Dementia. Clinical Psychology Review, Tarrytown, v. 9, p. 181-195, 1989.

ORANGE, J. B.; KERTESZ, Andrew. Discourse Analyses and Dementia. Brain and Language, New York, v. 71, n. 1, p. 172-174, 2000.

PARENTE, Maria Alice; CAPUANO, Andréa; NESPOULOUS, Jean-Luc. Ativação de Modelos Mentais no Recontar de Histórias por Idosos. Psicologia: Reflexão e Crítica, Porto Alegre, v. 12, n. 1, p. 157-172, 1999. 
PARENTE, Maria Alice et al. The Impact of the Interactivity of the Hypertext in the Recall of Aged and of Alzheimer's Patients. Revue Parole, Bruxelas, n. 29/30, p. 189-217, 2005.

PERRY, Richard; HODGES, John. Attention and Executive Deficits in Alzheimer's Disease: a critical review. Brain, London, v. 122, p. 383-404, 1999.

PIGNATTI, Ricardo et al. Specific Impairments of Selective Attention in Mild Alzheimer's Disease. Journal of Clinical and Experimental Neuropsychology, Lisse, v. 27, n. 4, p. 436-448, 2005.

SQUIRE, Larry; KANDEL, Eric. A Memória e as Bases Biológicas da Individualidade. In: . Memória: da mente às moléculas. Porto Alegre: Artmed, 2003. P. 211-232.

VAN DIJK, Teun. Cognição, Discurso e Interação. São Paulo: Contexto, 2004.

VIEIRA, André Guirland. Do Conceito de Estrutura: narrativa à sua crítica. Psicologia: Reflexão e Crítica, Porto Alegre, v. 14, n. 3, p. 599-608, 2001.

YESAVAGE, Jerome et al. Development and Validation of a Geriatric Depression Screening Scale: a preliminary report. Journal of Psychiatric Research, Oxford, v. 17, n. 1, p. 37-49, 1983.

Recebido em: 13-09-2007

$1^{a}$ revisão: $20-02-2008$

$2^{a}$ revisão: 09-06-2008

Aceite final: 13-06-2008 